\title{
A Virtual Instrument for Compressive Sensing of Multimedia Signals
}

\author{
Sanja Zuković, Milica Medenica, Andjela Draganić, Irena Orović, Srdjan Stanković \\ University of Montenegro, Faculty of Electrical Engineering, Džordža Vašingtona bb, 81000 Podgorica, Montenegro \\ Email:irenao@ac.me
}

\begin{abstract}
Compressive Sensing (CS) is currently a very popular signal acquisition approach. It provides an alternative way of signal sampling which is based on a small random set of measurements. The entire signal can be reconstructed from the measurements with high accuracy by using very complex mathematical algorithms if the certain conditions are met. Various algorithms for CS reconstruction have been proposed for different types of signals and different application requirements. In this paper, several commonly used algorithms for onedimensional (1D) and two-dimensional (2D) signals reconstruction are implemented within the Virtual Instrument for CS signals reconstruction. The Virtual Instrument is a userfriendly tool that provides efficient analysis of signals, using different algorithms and variety of options and parameters. It includes different multimedia test signals (both 1D and 2D signals), but also there is an option for user-defined signals.
\end{abstract}

Keywords - Compressive Sensing, reconstruction, $l_{1}$-norm minimization, Orthogonal Matching Pursuit, TV minimization

\section{INTRODUCTION}

Compressive Sensing/Sampling (CS) [1]-[4] is a recently developed approach for signal sampling and reconstruction. It allows a signal, having a sparse representation in certain domain, to be recovered from a small set of randomly acquired samples. Signal exhibits sparsity property if it can be represented by a small number of non-zero samples in the certain transform domain. The signal recovering procedure is based on minimization of different norms $\left(l_{0}, l_{1}, l_{2}\right.$ norm, etc.) [1]. The commonly used approach is based on $l_{1}$-norm minimization [5]-[7], solved by convex optimization algorithms. Another type of algorithms, called greedy algorithms (e.g. Orthogonal Matching Pursuit OMP, Gradient Pursuit GP [8]-[10], etc.) are based on iterative procedures. They provide much simpler and faster solutions, but may require some a priori knowledge about the signals. Generally speaking, these algorithms may provide different results depending on the signal nature, number of available measurements, or signal sparsity.

In this paper, the Virtual Instrument [11] for CS signal reconstruction is presented. Several algorithms for onedimensional (1D) and two-dimensional (2D) multimedia data reconstruction are implemented. Virtual Instrument allows user to choose between 1D and 2D signal reconstruction, as well as to test different algorithms and compare their performance. Additionally, the users may change the number of measurements and the domain of sparsity used in CS reconstruction. The reconstruction accuracy is quantified using different error types.

The paper is organized as follows. In Section II, the theoretical background on CS theory and reconstruction algorithms implemented in the Virtual Instrument is given. The overview of the Virtual Instrument properties and performance are given in Section III, while its functionality is presented in Section IV. Concluding remarks are given in Section V.

\section{THEORETICAL BACKGROUND}

\section{A. Compressive Sensing}

Signal reconstruction based on CS principles requires some conditions to be satisfied [2]-[4]. If we observe a signal $x$ of length $N$, it can be represented in terms of basis vectors $\psi_{i}$

(of size $N \times 1$ ):

$$
x=\sum_{i=1}^{N} s_{i} \psi_{i}=\psi s,
$$

where $s_{i}$ is $N \times 1$ column vector of transform domain coefficients. The first condition required in CS is sparsity, i.e., the signal should be sparse in the domain $\psi$. Furthermore, we assume that only $M$ measurements from $x$ are available and arrange them in $M \times 1$ vector $y$. Selection of the samples has to be done in a random manner, to provide incoherent measurements, as the second CS requirement. Matrix that selects $M$ out of $N$ samples is called measurement matrix, and is denoted by $\Phi(M \times N)$ :

$$
y=\Phi x \text {. }
$$

Further, from (1) and (2), $y$ can be written as:

$$
y=\Phi x=\Phi \psi s=\theta s,
$$

where $\theta=\Phi \psi$ is called CS matrix $(M \times N)$. The system of equations (3) is undetermined and can be solved using different optimization algorithms [5]-[10], [12]-[17].

\section{B. Algorithms for 1D Signals Reconstruction}

Some interesting optimization algorithms for 1D signals reconstruction will be described in the sequel. For instance, we focus to basis pursuit $l_{1}$-minimization method [5]-[7], [12], OMP [8]-[10], [12]-[13] algorithm and non-iterative thresholdbased algorithm [14]-[17]. 
1. $l_{1}$-minimization using primal-dual interior point method: In order to recover signal $x$ from its measurements $y=\Phi x$, the following optimization problem should be solved [2], [6]:

$$
\min \left\|s^{*}\right\|_{l_{1}} \text { subject to } y=\theta s^{*} \text {. }
$$

As $l_{1}$-norm is convex, linear programming can be used for solving this problem. The $s^{*}$ is sparse vector obtained as the result of the optimization.

2. OMP algorithm: Using the CS matrix $\theta$ and vector $y$, OMP approximate signal $s$ as linear combination of columns in $\theta$. In each iteration, the set of columns is expanded with additional column that best correlates with the residual signal. The algorithm can be summarized as follows:

1. Set the approximation error $r_{0}=y$, the initial solution to $s_{0}=0$ and $\mathrm{Z}_{0}=\varnothing$.

2. Do the following steps until the stopping criterion is met:

a) $Z_{n}=Z_{n-1} \cup \arg _{i} \max \left|\left\langle r_{n-1}, \theta_{i}\right\rangle\right|$,

b) $s_{n}=\underset{s}{\arg \min }\left\|r_{n-1}-Z_{n} s_{n-1}\right\|_{2}^{2}$, and $r_{n}=r_{n-1}-Z_{n} s_{n-1}$.

c) $n=n+1$ and $Z_{n}=Z_{n-1} \cup \arg _{i} \max \left|\left\langle r_{n-1}, \theta_{i}\right\rangle\right|$ until $n \leq K$, where $K$ is number of signal components.

\section{Non-iterative signal reconstruction solution}

The algorithm is based on the variances of estimation errors, calculated for each signal frequency [14]. The variance acts as a good indicator whether there is signal component on the observed frequency or not. Non-iterative algorithm provides fast reconstruction. It can be described as follows:

1 . For a given number of measurements $M$ on random positions $q_{M}$, calculate variance at each frequency:

$V(p)=\operatorname{var}\left(x\left(q_{M}\right) e^{-j 2 \pi p q_{M} / N}\right), p \in(1, N), N$ is signal length.

2. Find frequency positions $p_{0_{i}}$ that satisfy relation:

$$
p_{0_{i}}=\arg \min \{V(p)<\alpha \cdot \max V(p)\}, \text { for } p=1, \ldots, N,
$$

where $\alpha$ is $0.85 \leq \alpha \leq 0.95$.

3. Form CS matrix $\theta$ : matrix rows correspond to available measurements positions, while columns correspond to the frequencies $p_{0 \mathrm{i}}$.

4. Solve optimization problem: $\mathrm{X}=\left(\theta^{*} \theta\right)^{-1} \theta^{*} \mathrm{y}$

\section{Algorithms for $2 D$ Signals}

The image is not strictly sparse in any transform domain, but its gradient can be observed as a sparse signal. Thus, instead of standard algorithms, the Total Variation (TV) [18][20] minimization is used for the reconstruction of the 2D signals. The TV of signal $s$ represents the sum of the gradient magnitudes at each point $(i, j)$ :

$$
\|s\|_{T V}=\sum_{i, j}\left|(D s)_{i j}\right|,
$$

where $D$ represent operator described with relation:

$$
D_{i, j} s=\left[\begin{array}{l}
s(i+1, j)-s(i, j) \\
s(i, j+1)-s(i, j)
\end{array}\right] .
$$

Minimization problem with TV could be described as:

$$
\underset{s}{\min } T V(s) \text { subject to } y=\theta s
$$

It is important to note that all the implemented algorithms for 2D signals reconstruction are based on TV minimization procedure. The algorithms are shortly summarized below.

Algorithm 1 [19] takes samples from the 2D Fourier transform domain (2D DFT), along radial lines, and these samples will serve as measurements in the CS procedure.

Algorithm 2 [20] takes samples from 2D Discrete Cosine transform domain (2D DCT). Image is divided into blocks and the measurements are randomly taken from each block.

Algorithm 3 also uses 2D DCT as the observation domain. Small number of low-frequency DCT coefficients are used in this algorithm, while the rest of the measurement are randomly chosen pixels form middle and high frequency image regions.

\section{VIRTUAL INSTRUMENT}

The proposed Virtual Instrument is implemented in Matlab 7 , and provides implementation of CS algorithms including the efficient analysis of reconstructed signals. It is important to emphasize that various signals can be uploaded or defined using signal parameters. Also, the performance of the algorithms could be compared by observing error between original and reconstructed signal, as well as execution time. The outlook of the instrument is shown in Fig. 1. It consists of two parts: part for $1 \mathrm{D}$ and part for $2 \mathrm{D}$ signals reconstruction. Both parts contain the Definition and the Results blocks. The Definition part contains: the Signal choice block, the Percentage of measurements choice block (for 1D part), the Algorithm choice block and the Parameters choice block (for 2D part). The part Results has Numerical results block and Graphical results block.

In the Signal choice block, the user chooses different audio signals (in 1D part), or may choose between medical and natural images (in 2D part). Additionally, for audio signals the option to hear sounds of original and reconstructed signal is implemented as well. Definition of the new signals (number of components, amplitudes and frequencies) is provided within New signal block - Fig. 1. Both, 1D and 2D parts have Algorithm choice block where one of the algorithms described in the Section II, can be selected and applied. Numerical result block contains errors between original and reconstructed signal (MAE - Mean Absolute Error, MSE - Mean Squared Error) for 1D signal, and PSNR for the 2D signals. Algorithm execution time is shown in this block as well. Graphical result block includes original and reconstructed signal plots in chosen domain, while Graphical Error represents graphic representation of absolute error between original and reconstructed signal (with zooming option). 

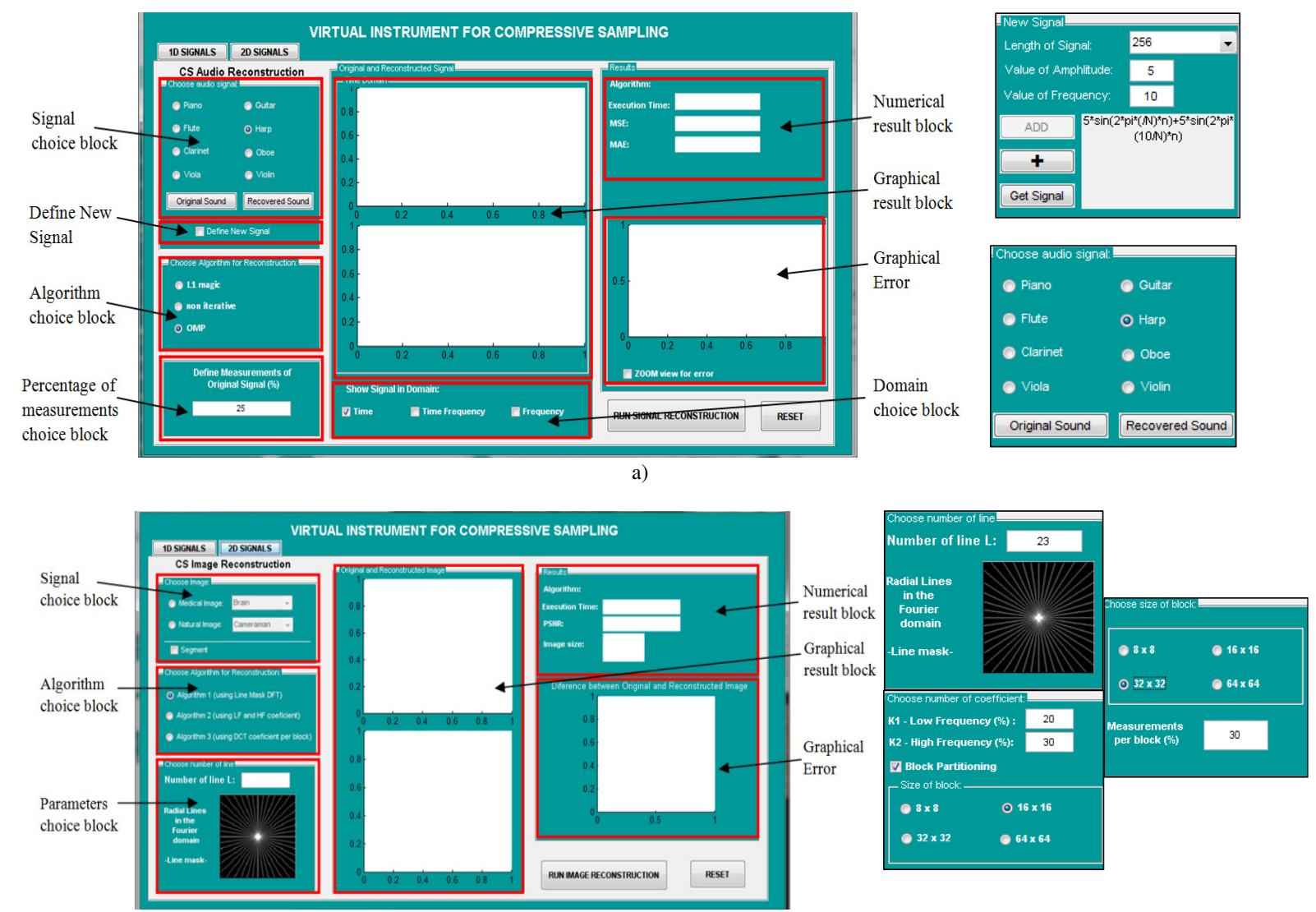

b)

Fig.1. The outlook of the proposed Virtual Instrument (for 1D and 2D signals)

Graphical Error part in 2D signals contains image obtained as a difference between original and reconstructed image. Percentage of measurements choice block allows user to define number of measurements, while Parameters choice block has in-built parameters, depending on the 2D reconstruction algorithm. In Domain choice block for 1D signal, user can choose in which domain signal to be appeared: time, time-frequency and frequency domain.

By pressing Run Reconstruction button, the recovered 1D or 2D signal will appear in the corresponding window. For the comparison, output will be at the same panel as input signal. The numerical result (MAE, MSE, PSNR, execution time) will be shown and allow user to easily make conclusions about the performance of the algorithms.

\section{FUNCTIONALITY OF VIRTUAL INSTRUMENT}

In this part the performances of the proposed Virtual Instrument are described on several signals. Firstly, 1D signals are observed.

\section{A. 1D signals reconstruction}

User can chose an audio signal representing a flute tone, for example. The observed signal is sparse in frequency domain and thus random samples are taken in time domain. In this example, the $40 \%$ of the randomly selected samples are taken for the reconstruction. Results obtained after running the $l_{1}$ reconstruction algorithm are shown in Fig. 2. Reconstruction quality for audio signal is measured by calculating MSE and MAE, as well as by plotting the MAE (Fig. 2). By comparing the sound of original and reconstructed signal, it has been confirmed that the quality of the reconstructed audio is preserved without introducing audible distortions. The $l_{1}$ reconstruction algorithm execution time for real audio signal is about $22 \mathrm{~s}$. Note that the execution time depends on the length of the signal, as well as on the chosen reconstruction algorithm.
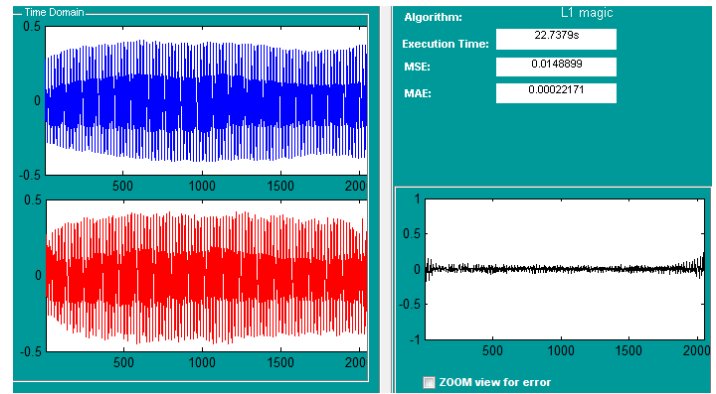

Fig. 2. Original (blue) and reconstructed (red) flute signal in time domain

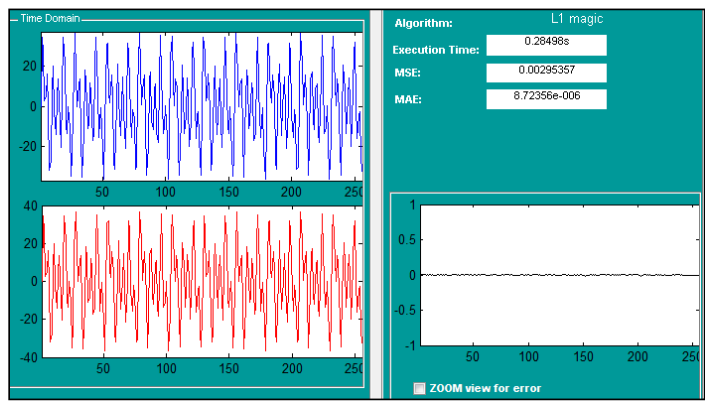

Fig. 3. Original and reconstructed user defined signal in time domain and reconstruction error 
A Virtual Instrument also allows user to specify the signal with parameters such as number of harmonics, value of amplitude and value of frequency, and analyze performance of different algorithms for the defined signal (Fig. 3). The quality of the reconstruction will be better and error will be smaller by using larger number of measurements. Observing results, user can conclude how many measurement of original signal should be taken, in order to obtain good quality of reconstruction.

\section{B. CS Image Reconstruction (2D Signals)}

Virtual Instrument implements some of the commonly used algorithms for image reconstruction and allows 2D signals analysis as well. The results using algorithm based on 2D DCT random measurements are shown in this example. The medical image "Brain" is chosen for reconstruction and the $30 \%$ of the total number of samples are used in CS procedure. Original and reconstructed images are shown in the Fig. 4 (left column). On the same panel, execution time is presented, as well as the value of PSNR. Difference between original and reconstructed signal is shown as error image in the same figure (right column of the Fig. 4).

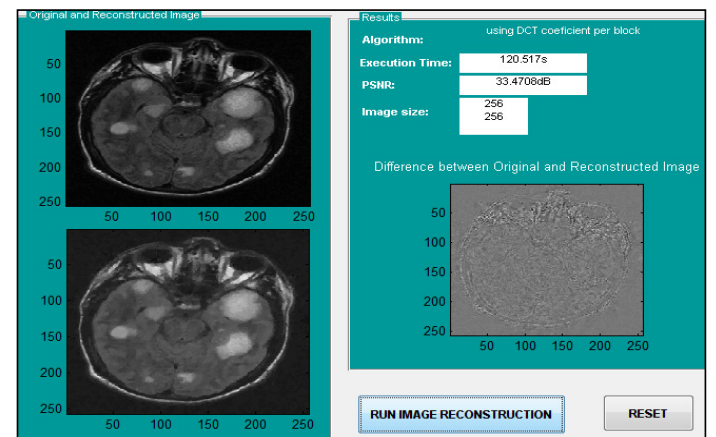

Fig. 4. Original and reconstructed image MRI Brain reconstructed by using Algorithm 3

\section{CONCLUSION}

The Virtual Instrument for CS signal reconstruction is proposed. It implements several algorithms for the reconstruction of $1 \mathrm{D}$ and $2 \mathrm{D}$ multimedia signals, such as audio signals and different types of images. The software is easy to use and provides a number of options for parameters settings and performance analysis. For instance, it has been shown that besides various CS algorithms, the users can change the number of measurements, as well as domain of sparsity. The proposed instrument provides the evaluation and comparison of different approaches (numerically and graphically). For all signal types, the algorithms execution time in sec is measured, as well. Therefore, the proposed Virtual Instrument could serve researches and practitioners in the CS field, and could be a very educative experimentation tool for those interested in CS for multimedia applications.

\section{ACKNOWLEDGMENT}

This work has been supported by the project CS-ICT (New ICT Compressive sensing based trends applied to: multimedia, biomedicine and communications), funded by Montenegrin Ministry of Science.

\section{REFERENCES}

[1] R. Baraniuk, "Compressive sensing," IEEE SP Magazine, vol. 24, no. 4 pp. 118-121, 2007.

[2] M. Elad, "Sparse and Redudant Representations: From Theory to Applications in Signal and Image Processing", Springer 2010.

[3] I. Orovic, S. Stankovic and T. Thayaparan, "Time-Frequency Based Instantaneous Frequency Estimation of Sparse Signals from an Incomplete Set of Samples," IET Signal Processing, Special issue on Compressive Sensing and Robust Transforms, May 2014.

[4] L. Stankovic, I. Orovic, S. Stankovic and M. Amin, "Compressive Sensing Based Separation of Non-Stationary and Stationary Signals Overlapping in Time-Frequency," IEEE Transactions on Signal Processing, vol. 61, no. 18, pp. 4562-4572, Sept. 2013

[5] D. L. Donoho and Y. Tsaig, "Fast Solution of $1_{1}$-norm Minimization Problems When the Solution May be Sparse", IEEE Transactions on Information Theory, vol. 54, no. 11, pp. 4789-4812, Nov. 2008

[6] S. Stankovic, I. Orovic and E. Sejdic, "Multimedia Signals and Systems," Springer-Verlag, New York, 2012.

[7] S. Stankovic, I. Orovic and M. Amin, "L-statistics based Modification of Reconstruction Algorithms for Compressive Sensing in the Presence of Impulse Noise," Signal Processing, vol.93, no.11, pp. 2927-2931, 2013

[8] S. Kunis and H. Rauhut, "Random Sampling of Sparse Trigonometric Polynomials, II. Orthogonal Matching Pursuit versus Basis Pursuit," Journal Foundations of Computational Mathematics archive, vol. 8, no. 6, pp. 737-763, Nov. 2008.

[9] J. Tropp and D. Needell, "CoSaMP: Iterative signal recovery from incomplete and inaccurate samples," Applied and Computational Harmonic Analysis, vol. 26, no. 3, pp. 301-321, May 2009.

[10] T. Blumensath and M. E. Davies, "Gradient Pursuits", IEEE Transactions on Signal Processing, vol. 56, no. 6, pp. 2370-2382, June 2008.

[11] I. Orovic, M. Orlandic, S. Stankovic and Z. Uskokovic, "A Virtual Instrument for Time-Frequency Analysis of Signals with Highly NonStationary Instantaneous Frequency," IEEE Transactions on Instrumentation and Measurements, vol. 60, no. 3, pp. 791 - 803, 2011.

[12] I. Orovic and S. Stankovic, "Improved Higher Order Robust Distributions based on Compressive Sensing Reconstruction," IET Signal Processing, May 2014.

[13] T. Zhang, "Sparse Recovery with Orthogonal Matching Pursuit Under RIP," IEEE Transactions on Information Theory, vol. 57, no.9, pp. 6215 $-6221,2011$.

[14] S. Stankovic, L. Stankovic and I. Orovic, "A Relationship between the Robust Statistics Theory and Sparse Compressive Sensed Signals Reconstruction," IET Signal Processing, Special issue on Compressive sensing and robust transforms, May 2014.

[15] L. Stankovic, S. Stankovic and M. Amin, "Missing Samples Analysis in Signals for Applications to L-estimation and Compressive Sensing," Signal Processing, vol. 94, pp. 401-408, 2014.

[16] S. Stanković, I. Orović and L. Stanković, "An Automated Signal Reconstruction Method based on Analysis of Compressive Sensed Signals in Noisy Environment", Signal processing, accepted for publication, May 2014.

[17] L. Stankovic, S. Stankovic, I. Orovic and M. Amin, "Robust TimeFrequency Analysis based on the L-estimation and Compressive Sensing," IEEE Signal Processing Letters, vol. 20, no. 5, pp. 499-502, 2013.

[18] J. Dahl, P. C. Hansen, S. H. Jensen and T. Li. Jensen, "Algorithms and software for total variation image reconstruction via first-order methods", Numerical Algorithms, vol. 53, no. 1, pp 67-92, 2010.

[19] X. Ye, Y. Chen, W. Lin and F. Huang, "Fast MR image reconstruction for partially parallel imaging with arbitrary k-space trajectories," IEEE Transactions on Medical Imaging, vol. 30, No. 3, pp. 575-85, 2011.

[20] J. Romberg, "Imaging via Compressive Sampling", IEEE Signal Processing Magazine, March 2008. 